\title{
Absorptionsanalyse der sternauslösenden Komponenten der Ultrastrahlung
}

\author{
Von E. Schopper, K. H. HöcKer, E. RösSLE \\ Aus der Forschungsstelle für Physik der Stratosphäre in der Max-Planck-Gesellschaft in \\ Weißenau und der Technischen Hochschule Stuttgart \\ (Z. Naturforschg. 6 a, 603-613 [1951]; eingegangen am 20. August 1951) \\ Erich Regener zum 70. Geburtstage
}

\begin{abstract}
Die Zahl der durch Ultrastrahlung in $2950 \mathrm{~m}$ Höhe hinter Blei-, Eisen- und Kohle-Absorbern ausgelösten Kernzertrümmerungen (Sterne) wird gemessen. In Blei und Eisen tritt ein Übergangseffekt der Sternhäufigkeit mit einer Zunahme um $35 \%$ bei $1,2 \mathrm{~cm}$ Blei bzw. $4,5 \mathrm{~cm}$ Eisen auf. Die beiden Maxima werden einer Sternauslösung durch Photonen zugeschrieben und der Anteil der durch Photonen in Luft erzeugten Sterne zu 10\% der Gesamthäufigkeit festgelegt. Diese Deutung ist verträglich mit dem experimentell bekannten Wirkungsquerschnitt der Sternauslösung durch Photonen $\left(5 \cdot 10^{-27} \mathrm{~cm}^{2}\right)$ und der Intensität der Photonenkomponente. Die Höhe, Lage und Form der Maxima ist in Übereinstimmung mit der aus der Kaskadentheorie errechneten Vermehrung. Weitere Maxima der Sternhäufigkeit werden bei $20 \mathrm{~cm}$ in Blei, bei $18-20 \mathrm{~cm}$ in Graphit der Dichte 2, festgestellt.
\end{abstract}

$\mathrm{D}$ ie Kern-Wechselwirkungen der kosmischen Strahlung in der Materie, die als Phänomen der "Sterne" in photographischen Platten oder in der Wilson-Kammer beobachtet werden, pflegt man einfacherweise einer global als N-Komponente bezeichneten Strahlung zuzuschreiben, ohne dabei die Frage nach ihrer Teilchennatur explicite zu berühren. Die formale Berechtigung dazu ergibt sich aus der in guter Näherung exponentiellen Abnahme der Sternhäufigkeit in der Atmosphäre oder hinter MaterieAbsorbern. Es ist heute bekannt, daß der überwiegende Anteil der beobachteten Sternhäufigkeit von energiereichen Nucleonen $\left(E>10^{9} \mathrm{eV}\right)$ und ihren sekundären Nucleonen $\left(E>1 / 2 \cdot 10^{8} \mathrm{eV}\right)$ gebildet wird. Jedoch ist die Beteiligung der übrigen in der kosmischen Strahlung enthaltenen Teilchen an der Erzeugung von Sternen im Verhältnis ihrer Häufigkeit und ihres jeweiligen Wirkungsquerschnittes für nucleare Wechselwirkungen sicher nicht vernachlässigbar. Wir haben in einer früheren rechnerischen Untersuchung ${ }^{1}$ versucht, auf Grund der experimentellen Daten der Intensitäten und Wirkungsquerschnitte die Beteiligung der einzelnen Komponenten der kosmischen Strahlung an der Sternerzeugung in verschiedenen Höhen zu ermitteln. Wir haben seinerzeit

1 K. H. H ö cker u. E. S ch o p per, Ann. Physik VI, 6, 338 [1949].

2 E. S chopper, K. H. H ö cker u. G. Kuhn, Physic. Rev. 82, 445, 446 [1951]; Physiker-Tagung Bad Nauheim, Oktober 1950.

3 G. K u h n, Diplomarbeit Stuttgart 1951.

4 W. Heitler, C. F. Powell u. H. He itler, Nature [London] 146, 65 [1940].
(1948/49) den Anteil der sekundären Nucleonen an der Sternauslösung aus ungenügender Kenntnis ihres Energie-Spektrums erheblich unterschätzt und waren gezwungen, die beobachteten Sternhäufigkeiten, besonders in den unteren Atmosphärenschichten, durch Hinzuziehung anderer Teilchen zu erklären.

Die damaligen Abschätzungen, die in dieser Hinsicht als überholt zu betrachten sind, veranlaßten uns $\mathrm{zu}$ einer eingehenden experimentellen Untersuchung der Beteiligung der einzelnen Komponenten an der Sternerzeugung durch eine Absorptionsanalyse der $\mathrm{N}$-Komponente in verschiedenen Materialien.

Wir haben im Herbst 1949 Absorptionsversuche mit elektronenempfindlichen Kernspurplatten (Kodak NT 4 und Iford G 5-Platten) aufgenommen, die wir auf der Zugspitze (2950 m über NN) hinter Absorbern von Blei, Eisen und Kohle (Graphit) exponierten ${ }^{2,3}$. Es lagen damals die Ergebnisse von $\mathrm{He}$ itler und Powell ${ }^{4}$ vor, die hinter Bleiabsorbern einen Übergangseffekt der Einzelspuren von Protonen bei $1-2 \mathrm{~cm}$ Bleidicke gefunden hatten, und die Arbeiten von $\mathrm{B}$ ernardini und Mitarbeitern ${ }^{5}$, mit einem ähnlichen Übergangseffekt für (energiearme) Sterne. Im Anschluß an Absorptionsmessungen von $\mathrm{Per}$ kin s ${ }^{6}$ hatten Ge orge und Jas o ${ }^{7}$ Sternhäufig-

5 G. B ernardini, G. Cortini u. A. M a nf re di n i, Nuovo Cimento 5, 511 [1948]; Cosmic Radiation, Bristol Sympos. 1948; Physic. Rev. 74, 845 [1948]; 76, 1792 [1949]; 79, 925 [1951].

6 D. H. P e rkin s, Nature [London] 160, 707 [1947].

7 E. P. G e o r g e u. A. C. J a s o n, Nature [London] 162, 333 [1948]; Proc. physic. Soc. 62, 243 [1949]; Cosmic Rad., Bristol Sympos. 1948. 
keiten hinter Blei und Kohle bis zu großen Absorberdicken gemessen, ohne allerdings bei dem großen Abstand ihrer Meßpunkte einen Übergangseffekt zu beobachten. (In der bis $200 \mathrm{~g} \mathrm{~cm}^{-2}$ gemessenen $\mathrm{Ab}$ sorptionskurve in Kohle ${ }^{7 \mathrm{a}}$ ist ein Maximum bei $50 \mathrm{~g} \mathrm{~cm}^{-2}$ entsprechend $20-25 \mathrm{~cm}$ Kohle angedeutet.) In der gleichen Richtung gingen Versuche von $\mathrm{H}$ arding, Lattimore, Li und Perkins ${ }^{8}$ mit Eis-Absorbern bis $5 \mathrm{~m}$ Dicke auf $3540 \mathrm{~m}$ Höhe. Einen eindeutigen Übergangseffekt der Sternhäufigkeit hinter Blei bei $1 \mathrm{~cm}$ Absorberdicke fanden L ord und $\mathrm{S} \mathrm{ch}$ e in ${ }^{9}$ in der Stratosphäre $(50 \mathrm{~mm} \mathrm{Hg})$, desgleichen berichten $\mathrm{Sh}$ apiro und Mitarbeiter ${ }^{9 \mathrm{a}}$ in einer kürzlich erschienenen Notiz über ein Maximum hinter $2 \mathrm{~cm}$ Blei und ein weiteres Maximum hinter $5-7 \mathrm{~cm}$, ebenfalls in der Stratosphäre.

Unsere eigenen Messungen ergeben ein eindeutiges Übergangsmaximum der Sternhäufigkeit hinter $1,2 \mathrm{~cm}$ Blei in Übereinstimmung mit obigen Beobachtungen ${ }^{4,5,9,9 a}$, und ein solches hinter $4,5 \mathrm{~cm}$ Eisen; wir ordnen sie einer Beteiligung von Photonen an der Sternerzeugung zu, die in Luft $10 \%$ der beobachteten Häufigkeit ausmacht. Darüber hinaus finden wir bisher ebenfalls nicht bekannte Maxima der Sternhäufigkeit hinter $20 \mathrm{~cm}\left(225 \mathrm{~g} \mathrm{~cm}^{-2}\right)$ Bleiabsorber und hinter $20 \mathrm{~cm}\left(40 \mathrm{~g} \mathrm{~cm}^{-2}\right)$ Kohleabsorber (Graphit) der Dichte 2. In Graphit der Dichte 1 beginnt ebenfalls ein Anstieg der Sternhäufigkeit bei etwa $15 \mathrm{~cm}$ mit flacherem Abfall.

\section{Experimentelle Ergebnisse}

Die prinzipielle Form der Blei- und Kohle-Absorber zeigt Abb. 1. In diesen Absorbern wurden in $\mathrm{Ab}$ ständen von $1 \mathrm{bzw} .2 \mathrm{~cm}$ Platten teils in horizontaler, teils in vertikaler Lage, gleichzeitig exponiert. Die Eisenabsorber waren Hohlkugeln von $20-35 \mathrm{~cm}$ Durchmesser mit verschiedener Wandstärke. Die Absorber waren in einer Holzbaracke einige Meter unter dem Gipfel des Zugspitz-Observatoriums in $2950 \mathrm{~m}$ über NN entsprechend $700 \mathrm{~g} \mathrm{~cm}^{-2}$ Luftschicht unter einem Holzdach von $5 \mathrm{~g} \mathrm{~cm}^{-2}$ Dicke untergebracht.

Mit jedem dieser Absorber wurden von 1949 bis 1951 mindestens drei Meßreihen durchgeführt mit Expositionszeiten von 20 bis 60 Tagen.

7a J. C. B arton, E. P. G e orge u. A. C. Juson, Proc. physic. Soc. (A) 64, 175 [1951].

8 J. B. Harding, S. Lattimore, T. T. Li u. D. H. Perkins, Nature [London] 163, 319 [1949]. [1949].

9 J. J. L ord u. M. S che in, Physic. Rev. 75, 1956

${ }_{9 a}$ M. Shapiro, B. Stiller, M. Birnbaum u.

F. W. O'D e ll, Physic. Rev. 83, 455 [1951] (Zusatz b. d. Korrektur).
Zur experimentellen Technik und zur Auswertung sei noch folgendes bemerkt: Für die Entwicklung der $400 \mu$ dicken Platten wurde ein Kalt-Warm-Zyklus ${ }^{10}$ angewendet ${ }^{11,3}$.

Der Auswertung der Platten liegt folgendes Schema zugrunde: Als Stern zählt jedes Ereignis mit mindestens zwei Spuren aus einem gemeinsamen Zentrum, von denen mindestens eine eine Länge $>70 \mu$ haben muß. Zweier-Sterne wurden gezählt, wenn Streuung einwandfrei ausgeschlossen war. Jeder Stern ist als schematisches Faksimile registriert, das die Kennzeichnung des auslösenden Teilchens (neutral oder ge-

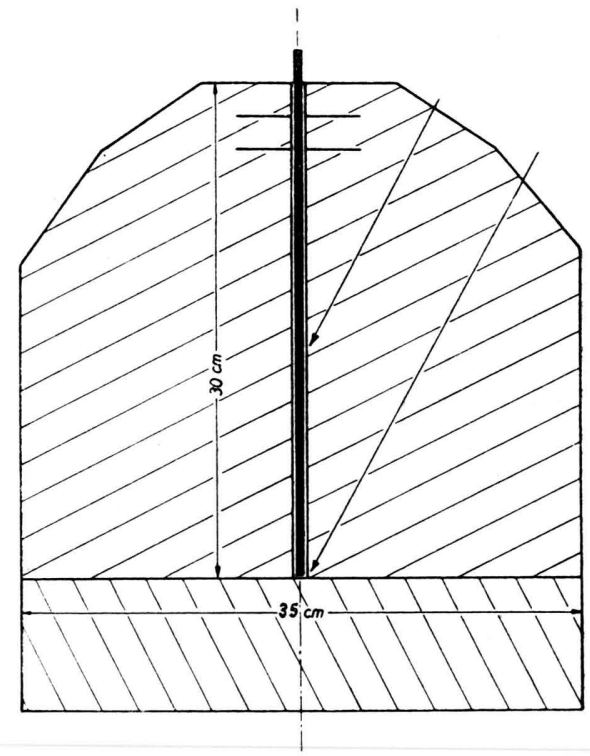

Abb. 1. Schematische Form des Blei- und Graphit-Absorbers.

laden) sowie der Spuren (schwarz, grau, relativistisch) enthält. Die durch negative $\pi$-Mesonen ausgelösten $\sigma$-Sterne sind gesondert gezählt.

a) Die Häufigkeit der Sterne mit $\geqq 2$ Spuren

Die Abb. 2, 3 und 4 geben die relative Häufigkeit aller Sterne mit mindestens 2 Spuren wieder, aufgetragen über der jeweiligen Dicke des Absorbers als Zusammenfassung der bisher ausgewerteten Messungen. Die Werte der jeweiligen Null-Platte der verschiedenen Meßreihen sind auf $100 \%$ normiert. Nicht mitgerechnet sind darin die $\sigma$-Sterne. Ohne Berück-

10 C. C. Dilworth, G. P. S. Occhialini u. R. N. P a y n e, Nature [London] 122, 102 [1948]. C. C. Dilworth, G. P. S. Occhialini u. L. Verm a e s e n, Bull. Centr. physic. Nucl. Brüssel 1950.

11 W. B r a u n, Diplomarbeit Stuttgart 1951. 
sichtigung der Zweier-Sterne ist der Kurvenverlauf derselbe, mit entsprechend geringerer Absoluthäufigkeit.

Die in den Abb. 2, 3, 4 eingezeichneten Kurven a, b, c, d stellen eine Zerlegung der gemessenen Sternhäufigkeit in die von verschiedenen Komponenten er-
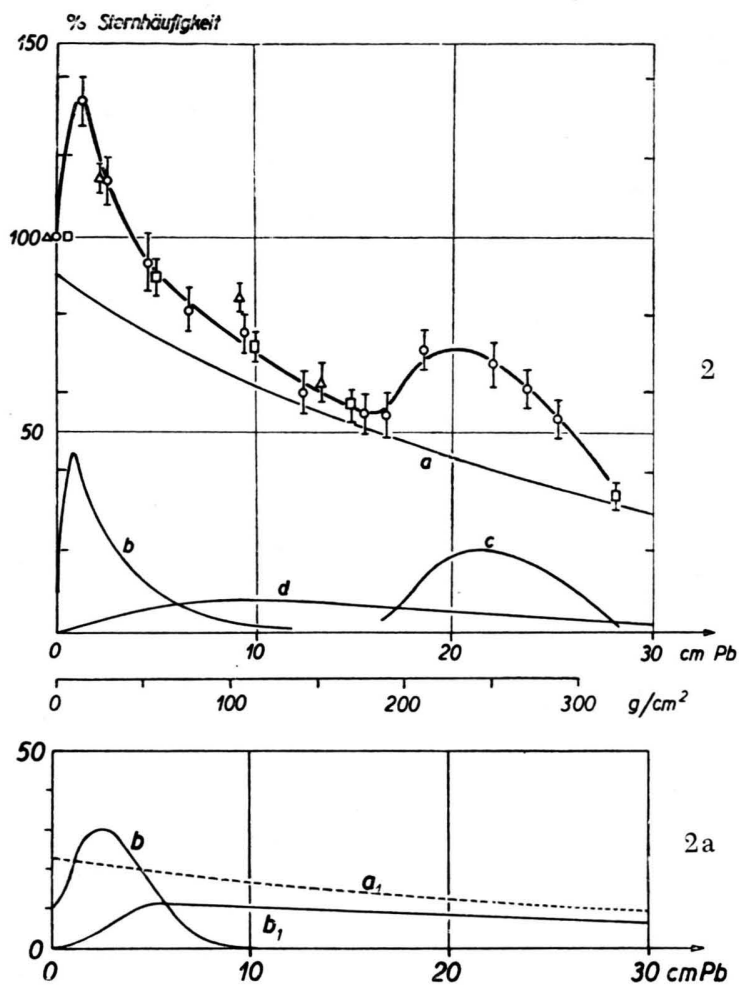

Abb. 2. Relative Sternhäufigkeit ( $\geqq 2$ Spuren) hinter Blei in $2950 \mathrm{~m}$ Höhe. $\circ \circ \circ$ eigene Messungen; $\square$ 口 Werte

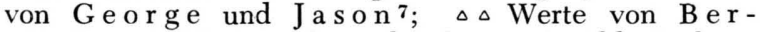
n a rdini und Mitarbb.5. Absorber nach Abb. 1; horizontale und vertikale phot. Platten. Kurven a, b, c, d Komponenten-Analyse, a = nuclearer Anteil der Sternhäufigkeit, b = Photonen-Anteil der Sternhäufigkeit, c, d siehe Text.

Abb. 2 a. Berechnete Komponenten-Analyse. a $a_{1}$ Sternanteil relativistischer Nucleonen, b Sterne aus Kaskadenphotonen, $b_{1}$ aus $\pi^{\mathbf{0}}$-Photonen.

zeugten Anteile dar, die wir im folgenden begründen werden. Kurve a repräsentiert den von der nuclearen Komponente ausgelösten Sternanteil, Kurve b den Anteil der photonausgelösten Sterne, der in Blei und Eisen zu Maxima bei 1,2 bzw. $4,5 \mathrm{~cm}$ führt (bMaxima). Die Kurven c entsprechen einer rein formalen Analyse der gemessenen Häufigkeitskurve nach Abzug der Komponenten ( $\mathrm{a}+\mathrm{b})$ (c-Maximum). In Blei ergibt sich dabei noch eine Restkomponente d, deren Verlauf und Größe bei unserer formalen Ana-
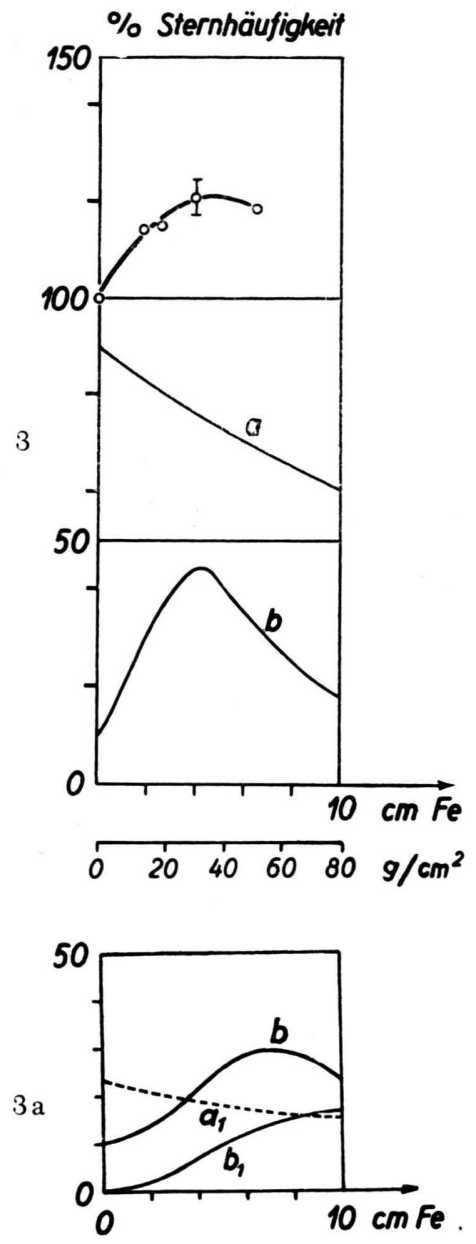

Abb. 3. Relative Sternhäufigkeit ( $\geqq 2$ Spuren) hinter Eisen in $2950 \mathrm{~m}$ Höhe. Kugelabsorber; Kurven a, b Komponenten-Analyse, $\mathrm{a}=$ nuclearer Anteil der Sternhäufigkeit, $\mathrm{b}=$ Photonen-Anteil der Sternhäufigkeit.

Abb. 3 a. Berechnete Komponenten-Analyse. a Sternanteil relativistischer Nucleonen, b Sterne aus Kaskadenphotonen, $b_{1}$ Sterne aus $\pi^{0}$-Photonen.

lyse jedoch nicht repräsentativ zu sein braucht. Wir geben weiter unten eine mögliche Deutung.

b) $\sigma$-S terne

An den Übergangseffekten sind die durch Einfang negativer $\pi$-Mesonen ausgelösten $\sigma$-Sterne, die in den Sternhäufigkeiten der Abb. 2, 3, 4 nicht enthalten sind, nur in geringem Maße beteiligt. Wir geben in Tab. 1 ihre Häufigkeiten hinter Blei und Graphit im Absolutbetrag an, zu vergleichen mit der Gesamthäufigkeit der übrigen Sterne ohne Absorber $z=14,4 \pm$ $0,7 / \mathrm{cm}^{3} \cdot d$. Bemerkenswert ist der beim Eintrit in 


\begin{tabular}{|c|c|c|}
\hline $\begin{array}{c}\text { Absorberdicke } \\
\mathrm{cm}\end{array}$ & \multicolumn{2}{|c|}{$\sigma$-Sterne $/ \mathrm{cm}^{3} \cdot d$} \\
\hline 0 & $\mathrm{~Pb}$ & $\mathrm{C}$ \\
\hline 1,2 & $0,147 \pm 0,03$ & $0,37 \pm 0,05$ \\
2,4 & $0,29 \pm 0,07$ & 0 \\
5,6 & $0,54 \pm 0,2$ & \\
10 & $0,52 \pm 0,2$ & $0,25 \pm 0,06$ \\
11,6 & $0,43 \pm 0,2$ & $0,61 \pm 0,15$ \\
18,6 & $0,54 \pm 0,2$ & $0,64 \pm 0,15$ \\
22,4 & $0,50 \pm 0,2$ & $0,62 \pm 0,15$ \\
\hline
\end{tabular}

Tab. 1. Absoluthäufigkeiten der $\sigma$-Sterne hinter Blei und Graphit in $2950 \mathrm{~m}$ Höhe.

den Kohle-Absorber beobachtete anfängliche Abfall der $\sigma$-Sternhäufigkeit, der statistisch gesichert ist.

e) Absorberform und Lage der Maxima

Die Idealform der Hohlkugel ließ sich aus Transportgründen nicht generell einhalten. Wir haben für die Blei- und Kohle-Messungen einige Kontrollpunkte mit Ideal-Absorbern gemessen. Für die anderen Absorber wurde unter Berücksichtigung der Winkelabhängigkeit der Einfallsintensität $I(\vartheta)$ der NKomponente eine möglichst gute geometrische Näherung ermittelt.

Wir betrachten von den einfallenden Nucleonen - die den Hauptanteil an der Sternauslösung ausmachen - zwei Gruppen:

1. Die erste umfaßt die energiereichen Nucleonen mit Energien $>5 \cdot 10^{8} \mathrm{eV}$. Nach den Messungen der B ristol-Gruppe ${ }^{12 a, d}$ und von $\mathrm{Harding}{ }^{13}$ an Sekundärteilchen aus Sternen in $3540 \mathrm{~m}$ Höhe besteht bei den energiereichen Nucleonen eine starke Bevorzugung der Richtung des auslösenden Nucleons. Wenn wir in großer Höhe $(x=0)$ die Intensität der Primärstrahlung richtungsisotrop annehmen, ergibt sich für die Höhe $x$ mit der Bezeichnung $I(x, 0)=I_{\text {v }}$ die gerichtete Intènsität

$$
I(x, \vartheta)=I_{\mathrm{v}} e^{-a x / \cos \vartheta},
$$

dabei ist $a^{-1}=138 \mathrm{~g} \mathrm{~cm}^{-2}$ die Absorptionslänge, die sich aus der Höhenabhängigkeit der Sternhäufigkeit in der Atmosphäre ergibt ${ }^{1,7,14-17}$.

12 a) R. H. Brown, U. Camerini, P. H. F ow le r, H. H e it le r u. D. T. K ing, Philos. Mag. J. Sci. 40, 862 [1949]. b) U. C a merini, T. C o or, J. H. Davies, P. H. Fowler, W. O. Lock, H. Mu ir he ad u. N. Tobis, Philos. Mag. J. Sci. 40, 1073 [1949]. c) P. H. F ow le r, Philos. Mag. J. Sci. 41, 169 [1950]. d) U. Camerini, P. H. Fowler, W. O. L o ck u. H. Muirhe ad, Philos. Mag. J. Sci. 41, 413 [1950]. e) A. G. Carlson, J. E. Hooper u. D. T. K ing, Philos. Mag. J. Sci. 41, 701 [1950].
Mit dieser Richtungsverteilung, die nach Deutsch mann ${ }^{18}$ auch die ausgedehnten Luftschauer auf Meereshöhe zeigen, wird die Zahl der Sterne $n(\vartheta) \mathrm{d} \vartheta$, die in der Flächeneinheit einer horizontalen photographischen Platte der Emulsionsdicke $D$ mit dem Wirkungsquerschnitt $Q$ durch die über das gesamte Azimut aus dem Zenitwinkel d $\vartheta$ einfallende Strahlung erzeugt werden

$$
\begin{gathered}
n(\vartheta) \mathrm{d} \vartheta=2 \pi I_{\mathrm{v}} N D Q e^{-a x / \cos \vartheta} \sin \vartheta \mathrm{d} \vartheta \\
\left(N=\text { Zahl der Atome } \cdot \mathrm{cm}^{-3}\right) .
\end{gathered}
$$

Die Funktion hat für $\vartheta=25^{\circ}$ ein Maximum; der Hauptanteil der durch energiereiche Nucleonen aus-

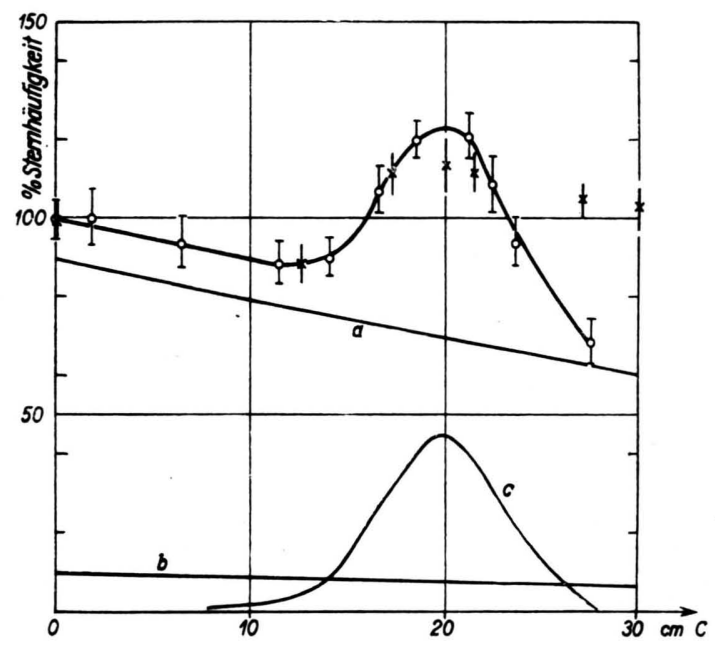

Abb. 4. ○ Relative Sternhäufigkeit $\geqq 2$ Spuren) hinter Kohlenstoff in $2950 \mathrm{~m}$ Höhe. Absorber nach Abb. 1 (0 bis $35 \mathrm{~cm}$, Dichte $\varrho=2 \mathrm{~g} \mathrm{~cm}^{-3}$. Kurven a, b, c Komponenten-Analyse. $\mathrm{a}=$ nuclearer Anteil der Sternhäufigkeit, $\mathrm{b}=$ Photonen-Anteil der Sternhäufigkeit, $\mathrm{c}$ siehe Text. x x Relative Sternhäufigkeit hinter Kohlenstoff in $2950 \mathrm{~m}$ Höhe. Absorber nach Abb. $1(0-45 \mathrm{~cm}$, Graphitpulver $\left.\varrho=1 \mathrm{~g} \mathrm{~cm}^{-3}\right)$.

gelösten Sterne wird demnach durch Strahlungsanteile aus diesem Zenitwinkel gebildet. Für vertikal liegende Platten gilt eine analoge Abschätzung.

2. Die zweite Gruppe enthält die energieärmeren Nucleonen $5 \cdot 10^{7}<E<5 \cdot 10^{8} \mathrm{eV}$, die noch Sterne auszulösen vermögen und in der photographischen Platte die sogenannten ,grauen“ Spuren bilden. So-

13 J. B. H a r d i n g, Philos. Mag. J. Sci. 42, 63 [1951]. 14 M. T e u cher, Physiker-Tagung in Frankfurt, April 1951; Physik. Verhandl. 3, 1951.

15 B. R os s i, Rev. mod. Physics 20, 537 [1948].

16 H. Y a g o d a , Proc. Echo Lake Conf. 169, 1949.

17 E. O. S a l a n t, J. Horn bostel u. E. M. Dollm a n n, Physic. Rev. 74, 694 [1948].

18 M. Deuts chmann, Z. Naturforschg. 2a, 61 [1947]. 
weit sie aus mehrspurigen Sternen hervorgehen, ist ihre Winkelverteilung weitgehend isotrop ${ }^{12: a}$. Daneben finden $\mathrm{L}$ a t t i m or $\mathrm{e}^{19}$ sowie $\mathrm{Cu}$ ër und $\mathrm{Mo}$ r a $\mathrm{d}^{20}$ bei der Untersuchung der Richtungsverteilung von Einzelprotonen einen erheblichen Anteil von Teilchen, die in der photographischen Platte sich um den Zenitwinkel $\vartheta \sim 30^{\circ}$ gruppieren.

Wir können als eine angemessene Mittelung eine $\cos ^{2}$-Verteilung ${ }^{21,}{ }^{22}$ zugrunde legen. Dann ergibt sich an Stelle von (2)

$$
n(\vartheta) \mathrm{d} \vartheta=2 \pi I_{\mathrm{v}} N D Q \cos ^{2} \vartheta \sin \vartheta \mathrm{d} \vartheta .
$$

Die Verteilungsfunktion (3) ist als Polardiagramm in Abb. 5 dargestellt. Der Hauptanteil der Sterne wird aus Strahlungsrichtungen $\vartheta=35^{\circ}$ gebildet. Dieser

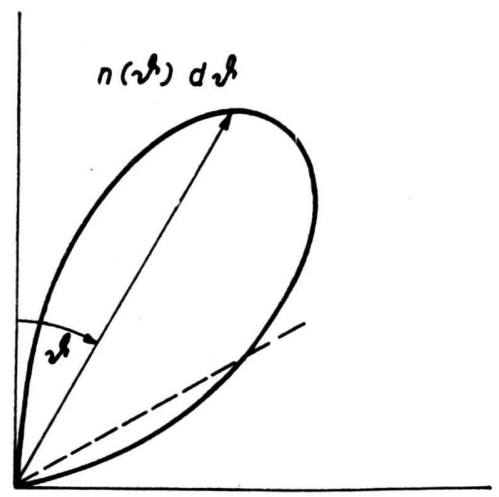

Abb. 5. Relative Sternhäufigkeit $n(\vartheta) \mathrm{d} \vartheta$, erzeugt in einer horizontalen photographischen Schicht durch die aus dem Zenitwinkelelement $\vartheta, \vartheta+\mathrm{d} \vartheta$ einfallende N-Strahlung.

Verteilung ist die Form unserer Absorber gut angepaßt. Die vertikale Schichtdicke des Absorbers ist entsprechend auf eine effektive umzurechnen.

Wir haben bei den einzelnen Meßreihen die Form und die Tiefe der Blei- und Graphit-Absorber teilweise geändert, um festzustellen, ob ein Einfluß der Absorberform auf die Übergangskurve vorliegt. Ein Meßpunkt des b-Maximums bei $1 \mathrm{~cm}$ Bleidicke z. B. wurde durch einen Ideal-Absorber kontrolliert; er liegt auf der mit dem Absorber nach Abb. 1 gemessenen Kurve.

Eine gute Übereinstimmung der Lage unseres bMaximums ist vorhanden mit dem von Lord und

19 S. L a t t i m or e, Philos. Mag. J. Sci. 40, 394 [1949] und 41, 819 [1950].

20 M. M or and u. P. Cuër, Cosmic Rad., BristolSymp. 1948.

21A. C. C o a te s u. R. H. He rz, Philos. Mag. J. Sci. 40, 1088 [1949].

22 N. P a g e, Proc. physic. Soc. 63, 250 [1950].
S che in ${ }^{9}$ in der Stratosphäre mit einem ähnlichen Blei-Absorber gemessenen Übergangsmaximum der Sternhäufigkeit (Abb. 6).

Für das c-Maximum in Blei finden wir denselben Kurvenverlauf mit einem 22, 27 und $30 \mathrm{~cm}$ tiefen Absorber. Eine geringe Verschiebung des c-Maximums tritt bei einer Änderung der Absorber-Breite auf, die sich als Änderung der effektiven Absorbertiefe für schräg einfallende Strahlung auswirkt.

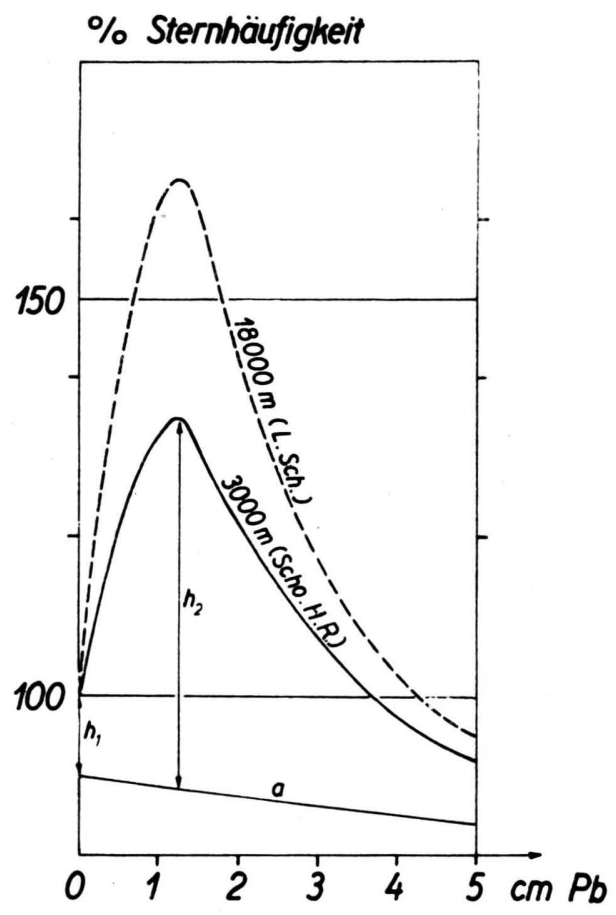

Abb. 6. Übergangseffekt der Sternhäufigkeit hinter Blei. L ord und S che in 9 , $18000 \mathrm{~m}$ (Sterne mit $\geqq 3$ Spuren); $\longrightarrow$ S chopper, Höcker, Rößle, 2950 m (Sterne mit $\geqq 2$ Spuren).

Der Kohle-Absorber bestand aus Klötzen von gepreßtem Graphit der Dichte 2. Bei der letzten Meßreihe haben wir noch einen zweiten affin vergrößerten Kohle-Absorber, bestehend aus Graphitpulver der Dichte 1, verwendet, mit einer Gesamttiefe von $45 \mathrm{~cm}$. In diesem Absorber beginnt der Anstieg des $c$-Maximums ebenfalls bei $15 \mathrm{~cm}$, ohne den bei Graphit der Dichte 2 beobachteten steilen Abfall (Abb. 4 , $x$-Meßpunkte).

Wir haben die Beobachtung gemacht, daß bei manchen - nicht bei allen - Meßreihen, sowohl in Blei wie auch in Eisen und Kohle, die Sternhäufigkeit der Nullpunkte um $20-30 \%$ gegenüber dem generellen Kurvenverlauf zu hoch liegt. Der Effekt tritt 
besonders dann auf, wenn die Nullplatte in einiger Entfernung vom Absorber frei im Raum in der Nähe der Holzdecke hängt. Das schließt es aus, daß er durch zusätzliche Sternauslösung durch rückgestreute Sekundärnucleonen aus dem Absorber verursacht ist. Man erhält einen zu steilen Abfall der Anfangskurve. Im Hinblick auf die Übergangseffekte in Kohle kann eine zusätzliche Materieschicht über dem Absorber bei unseren $\mathrm{zu}$ verschiedenen Jahreszeiten durchgeführten Messungen, z. B. eine gelegentliche, nicht ganz vermeidbare Schneelage auf dem Holzdach der Baracke - einen solchen Effekt verursachen.

Solange wir eine eindeutige Zuordnung noch nicht haben, haben wir hier für diese Diskussion die betreffenden Messungen nicht mitverwendet. Shapiro und Mitarbeiter ${ }^{9 \mathrm{a}}$ finden in der Stratosphäre in Bleiabsorbern bei Dicken von $0-1,5 \mathrm{~cm}$ einen solchen Abfall ebenfalls.

\section{d) Das Teilchenzahl-Spektrum der Sterne}

Bei ihren Absorptionsmessungen hinter Blei und Kohle in $3400 \mathrm{~m}$ Höhe fanden George und J a s o ${ }^{7}$ eine von der Tiefe des Absorbers unabhängige Verteilung der Teilchenzahl der Sterne (aller sichtbaren Spuren) von der Form

$$
N(>n)=K N_{0} e^{-\xi n},
$$

wobei $n$ die Teilchenzahl (Protonen) je Stern bedeutet und

$$
\begin{array}{ll}
K=1, & \xi=0,48 \text { für } 3<n<9 \\
K=0,28, & \xi=0,33 \text { für } n \geq 9 \text { ist. }
\end{array}
$$

Das Teilchenzahl-Spektrum der Sterne hängt, nach den Versuchen mit künstlich beschleunigten Teilchen $\alpha, \mathrm{p}, \mathrm{d}, \gamma)$ von der auslösenden Strahlung ab. Es besteht empirisch ein Zusammenhang zwischen der Teilchenzahl des Sterns und der auf den Kern übertragenen Anregungsenergie ${ }^{12 a}$. Insofern schlossen $\mathrm{G}$ e or ge und $\mathrm{J}$ a s o $\mathrm{n}^{7}$ bei ihren Messungen aus de: Konstanz des Tz.-Spektrums auf eine unveränderte Qualität der auslösenden N-Komponente hinter Blei.

Wir finden übereinstimmend mit den genannten Autoren ein konstantes Tz.-Spektrum für die Sterne hinter $0-4,6-6,6-8,6-10,6-12,6-15,6 \mathrm{~cm}$ Blei (Abb. 7, Kurve a). Die Sterne hinter 1,2 und 2,4 cm

23 J. B. H a r ding, Nature [London] 163, 440 [1949]; J. B. H a rding, S. Lat $\mathrm{t}$ imore u. D. H. Perkins, Proc. Roy. Soc. [London] Ser. A 196, 325 [1949].
Blei (Kurve b) sind etwas teilchenärmer. Der Anteil neutral ausgelöster Sterne ist ebenfalls höher. Eine auffallende Verschiebung des Teilchenzahl-Spektrums zu geringeren Teilchenzahlen finden wir im c-Maximum (Kurve c).

Das Teilchenspektrum der Sterne ist andererseits abhängig vom zertrümmerten Kern ${ }^{22-26}$. Der Exponent $\xi$ in (4) fällt mit zunehmender Nucleonenzahl etwa proportional $A^{-1 / 326}$. Dies macht sich in unserer

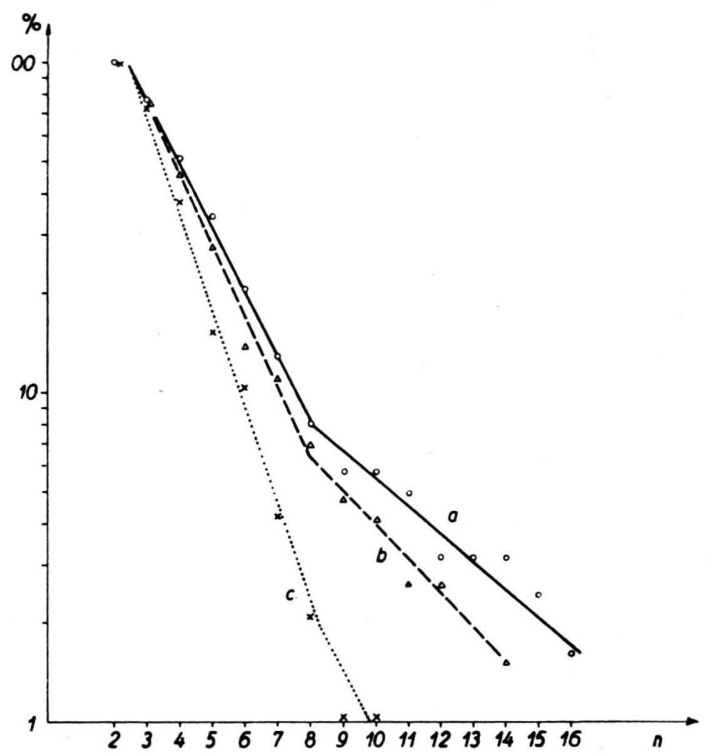

Abb. 7. Teilchenzahl-Spektrum der Sterne $(\alpha-\mathrm{u}$. Protonenspuren) hinter Blei. Kurve a normale Verteilung; b im bMaximum $(1,2 \mathrm{~cm} \mathrm{~Pb})$; c im c-Maximum $(20 \mathrm{~cm} \mathrm{~Pb})$.

obigen Verteilung natürlich nicht bemerkbar, da wir stets die in der Emulsion der Photoplatte ausgelösten Sterne beobachten: die Sterne sind bei uns Indikator der hinter dem Absorber vorhandenen sternauslösenden Strahlung. Dagegen ist dieser Einfluß in bezug auf die Sekundärnucleonen zu beachten, die aus den im Absorber erzeugten Sternen stammend, die Photoplatte erreichen ${ }^{2}$.

\section{Analyse der Absorptionskurven}

Bei der Analyse der Absorptionskurven gehen wir zunächst von der nuclearen Komponente aus, da empirisch bekannt ist, daß sie den Hauptanteil der Sterne liefert.

24 J. B a rbour u. L. Greene, Physic. Rev. 79, 406 [1950].

25 M. G. K. M en on, H. M u irhead u. O. Rochat, Philos. Mag. J. Sci. 41, 583 [1950].

26 P. E. H o d g s o n, Philos. Mag. J. Sci. 42, 82 [1951]. 
Aus der bekannten Materialabhängigkeit ihrer Absorption in der Luft $\left(a_{\mathrm{L}}^{-1}=138 \mathrm{~g} \mathrm{~cm}^{-2}\right)$, Blei $\left(a_{\mathrm{Pb}}^{-1}=\right.$ $\left.310 \mathrm{~g} \mathrm{~cm}^{-2}\right)$, Eisen $\left(a_{\mathrm{Fe}}^{-1}=200 \mathrm{~g} \mathrm{~cm}^{-2}\right)$ folgt sofort, daß Nucleonen für die b-Maxima bei $1,2 \mathrm{~cm}$ Blei und $4,5 \mathrm{~cm}$ Eisen nicht verantwortlich sein können. Ihre Form und Lage spricht für die Elektron-PhotonKomponente. Elektronen können nach unseren Beobachtungen als geladene auslösende Teilchen ausgeschlossen werden; außerdem ist ihr experimentell gemessener Wirkungsquerschnitt für Kernwechselwirkungen von der Größenordnung $10^{-29} \mathrm{~cm}^{2}$. Für Photonen dagegen ist der experimentelle Nachweis der Sternauslösung mit einem Wirkungsquerschnitt von einigen $10^{-27} \mathrm{~cm}^{2}$ durch Messungen von $\mathrm{K} \mathrm{i} \mathrm{k} \mathrm{u} \mathrm{ch} \mathrm{i}{ }^{27}$ erbracht. Wir werden im Detail die Anteile der nuclearen und der Photonenkomponente an der Sternauslösung durch Vergleich der Kurven in Blei, Eisen und Kohle analysieren und die Deutung der bMaxima als Photonen-Maxima rechnerisch begründen.

Für die c-Maxima in Blei und Kohle können wir zur Zeit lediglich den experimentellen Befund mitteilen. Eine Deutung ist in Anbetracht des steilen Anstiegs mit den bekannten Wechselwirkungsmechanismen der verschiedenen Elementarteilchen kaum möglich. Wir begegnen hier demselben typischen Verlauf, der auch beim zweiten und dritten Maximum der Rossi-Kurve gefunden und durch Messungen von $\mathrm{B}$ othe und Thurn ${ }^{28}$ und von Clay ${ }^{29}$ und Mitarbeitern in der letzten Zeit erneut bestätigt worden ist.

1. Die Absorption der nuclearen Komponenteundihr Anteilander Sternhäufigkeit

Wir haben früher ${ }^{2}$ die Teilchen der nuclearen Komponente in drei Gruppen eingeteilt: Eine energiereiche Gruppe relativistischer Protonen und Neutronen $\left(E>10^{9} \mathrm{eV}\right)$, eine mittlere Gruppe energieärmerer Nucleonen $\left(1 / 2 \cdot 10^{8}<E \leqq 10^{9} \mathrm{eV}\right)$ und schließlich die Nucleonen der dritten Gruppe $(E<$ $1 / 210^{8} \mathrm{eV}$ ). Die untere Grenze der zur Auslösung eines Sternes ( $\geqq 2$ Spuren) notwendigen Energie liegt bei 30 bis $50 \mathrm{MeV}^{1,30}$. Im wesentlichen sind also die Nucleonen der ersten und zweiten Gruppe für die Sternauslösung verantwortlich.

27 S. Kiku chi, Physic. Rev. 81, 1060 [1951].

28 W. B othe u. H. Thurn, Physic. Rev. 79, 544 [1950]; Diskussionskonferenz Kernphysik-Ultrastrahlung, Heidelberg 1951; Z. Naturforschg. 6a, 576 [1951].

29 J. Cla y u. W. L. S che en, Physica 14, 489 [1942]. J. C l a y u. G. K le i n, Physica 16, 293 [1950]. J. C l a y, Rev. mod. Physics 21, 90 [1949].

\begin{tabular}{|l|c|c|c|}
\hline Absorber & $\begin{array}{c}\text { Gerechnet } \\
\text { prop. } A^{1 / 3}\end{array}$ & Gemessen & Literatur \\
\hline Luft & 130 & 130 & 14 \\
& & 135 & 5 \\
$\mathrm{C}$ & 122 & $160 \pm 8$ & 7 \\
$\mathrm{Fe}$ & 200 & $143 \pm 10$ & 7 \\
$\mathrm{~Pb}$ & 310 & $360 \pm 60$ & $7 \mathrm{a}$ \\
$\mathrm{Phot.}$ & & $310 \pm 10$ & 5 \\
Emulsion & 220 & & 7 \\
\hline
\end{tabular}

Tab. 2. Absorptionslängen $\left(\mathrm{g} \mathrm{cm}^{-2}\right)$ der $\mathrm{N}$-Komponente in verschiedenen Absorbern.

Der Wirkungsquerschnitt für die Zertrümmerung eines Kernes durch relativistische Nucleonen ist proportional zu dessen Größe $\Sigma_{\mathrm{K}}=\Sigma_{0} A^{2 / 3}$, mit $\Sigma_{0}=$ $8 \cdot 10^{-26} \mathrm{~cm}^{2}$. Dem entspricht eine freie Weglänge $\lambda_{\mathrm{K}}=\lambda_{0} A^{1 / 3}$, in Luft $\sim 50 \mathrm{~g} \mathrm{~cm}^{-2} 31-33$. Mit solchen Kernwechselwirkungen ist durchweg Mesonenerzeugung verbunden. Die Absorption des Nucleons erfolgt nicht katastrophisch. Man rechnet in Luft im allgemeinen 2-3 Kernwechselwirkungen auf die Absorptionslänge $\left(a^{-1}\right)$, die empirisch ebenfalls einem $A^{1 / 3}$-Gesetz entspricht: $a^{-1}$ prop $A^{1 / 3}$. Für die energieärmeren Nucleonen der zweiten Gruppe $\left(1 / 2 \cdot 10^{8}<\right.$ $E \leqq 10^{9} \mathrm{eV}$ ) nimmt der Wirkungsquerschnitt (WQ) für die Erzeugung von Mesonen stark ab und beträgt bei einigen $10^{8} \mathrm{eV}$ wenige Prozent des obigen Wertes. Jedoch bleibt ein sehr viel größerer WQ für Kernzertrümmerungen bestehen. Man kann in den mittleren Atmosphärenschichten mit einem Gleichgewicht zwischen den Nucleonen der ersten und zweiten Gruppe rechnen, da letztere aus den von der ersten Gruppe erzeugten Sternen stammen. Für die zweite Gruppe ist experimentell dieselbe Absorptionslänge festgestellt ${ }^{5-8,12,14,19,33}$.

Wir haben in Abb. 2, 3, 4 den a-Kurven des nuclearen Anteils an der Sternauslösung die gerechneten, auf die Bleiabsorber mit $a_{\mathrm{Pb}}^{-1}=310 \mathrm{~g} \mathrm{~cm}^{-2}$ normierten Absorptionslängen von Tab. 2 zugrunde gelegt, mit der Voraussetzung, daß $90 \%$ der Sterne in Luft durch Nucleonen ausgelöst sein sollen.

Das $A^{1 / 3}$-Gesetz für die Absorptionslänge der $\mathrm{Nu}$ cleonen bedeutet eine in Blei und Eisen flachere Absorptionskurve gegenüber Kohle und Luft; sie ist für

30 J. B. H a r d in g, Philos. Mag. J. Sci. 40, 530 [1949],

31 G. C o c c o n i, Physic. Rev. 75, 1074 [1949].

32 J. T in lot, Physic. Rev. 74, 1197 [1948].

33 L. B a jalà, M. Merlin u. G. Puppi, Nuovo Cimento 7, 525 [1950]. 
die Sternerzeugung hinter Blei und Kohle und Eis als Generallinie bestätigt ${ }^{6-8,14}$.

Neben diesem allgemeinen Effekt können Übergangseffekte durch veränderte Erzeugung von Sekundären entstehen. Das Spektrum der Sekundär-Nucleonen ist verschiedentlich gemessen worden (vgl. z. B. 12, 13, 19, 23, 26), bislang jedoch entweder an Einzelspuren, die von der Atmosphäre her oder aus dünnen Absorberschichten die Photoplatte erreichen, oder an Sternen, die in der Photoplatte ausgelöst werden.

Es wäre von größtem Interesse, Intensitäten, Energie- und Teilchenspektrum der aus verschiedenen $\mathrm{Ab}$ sorbern kommenden Sekundärnucleonen in verschiedener Tiefe derselben zu kennen. Diese sehr langwierige Messung haben wir bisher noch nicht durchgeführt. Mes s el ${ }^{34}$ hat die Entstehung der Sekundärnucleonen in Luft über den Mechanismus einer Nucleonen-Kaskade in Kernmaterie rechnerisch behandelt. Das Ergebnis einer solchen Rechnung hängt naturgemäß stark von den Annahmen über den Entstehungsmechanismus der Sekundären ab. Gerade für die in Frage kommenden energiereichen Sekundären ist dieser aber noch unklar ${ }^{5}$. Für energiearme Nucleonen der 3. Gruppe (durch Neutronen in der Photoplatte erzeugte Anstoßprotonen) haben wir einen Übergangseffekt in Blei experimentell gefunden ${ }^{2}$.

Nach den Messungen von $\mathrm{Hodgs}$ on ${ }^{26}$ gilt für das Teilchenzahlspektrum der Verdampfungsnucleonen („schwarze“ Spuren in der Photoplatte) von Sternen, die in $3000 \mathrm{~m}$ Höhe durch Ultrastrahlung an Kernen einheitlicher Massenzahl ausgelöst sind:

$$
\begin{aligned}
& N(n) \mathrm{d} n=\text { const } e^{-n} \xi \mathrm{d} n, \\
& \text { wobei } \xi=\xi(A) \text { prop. } A^{-1 / 3} \text { ist }^{22-26} \\
& \text { und }=0,6 \text { für } \mathrm{C}, \mathrm{O}, \mathrm{N} \text {-Sterne, } \\
& =0,25 \text { für } \mathrm{Ag}, \mathrm{Br}-\text { Sterne, } \\
& =0,18 \text { für } \mathrm{Pb} \text {-Sterne. }
\end{aligned}
$$

Danach ist für diese Nucleonengruppe die Multiplizität je Stern in Blei höher als in Luft. Sie sind aber nicht mehr zur Auslösung von Sternen fähig. In der Stratosphäre, wo eine relativ große Zahl energiereicher Nucleonen vorhanden ist, kann das von $\mathrm{S}$ h a p ir ${ }^{9 \mathrm{a}}$ beobachtete Maximum der Sternhäufigkeit bei 6-7 cm Blei einen solchen nuclearen Über-

34 H. Messe 1, Commun. Dublin Inst. Advanced Physics (A) 7, 1951; Proc. Roy. Irish Soc. (A) 54, 8, 16 [1951].

35 N. Dallaporta, M. Merlin u. G. Puppi, Nuovo Cimento 7, 99 [1950]. gangseffekt darstellen. Für Gebirgshöhen haben Dallaporta, Puppi und Merlin ${ }^{35}$ einen schwachen nuclearen Übergangseffekt auch für Sterne in Blei errechnet. Sein Vorhandensein in $2950 \mathrm{~m}$ Höhe können wir unseren Messungen nicht mit Sicherheit entnehmen. Er kann in Kurve d (Abb. 2) enthalten sein. Dagegen halten wir es für unmöglich, die b-Maxima unserer Kurven in Abb. 2 und 3 durch einen nuclearen Übergangseffekt zu erklären. Über die c-Maxima können wir im Augenblick noch nichts aussagen.

\section{Die Photonenkomponente}

Die weitere Analyse unserer Absorptionskurven in Blei und Eisen in der Umgebung des ersten Maximums führen wir mit der Annahme durch, daß die Sternhäufigkeit in Luft, vor Eintritt der Strahlung in Blei, zu 90\% durch Nucleonen (Kurve a in Abb. 2, 3, $4,6)$ bewirkt sei, während $10 \%$ auf Photonen entfallen. Diese Abschätzung ist durch zwei Forderungen eingegrenzt. Einmal muß die Intensität der Photonen in $3000 \mathrm{~m}$ Höhe mit dem von $\mathrm{K} \mathrm{i} \mathrm{k} \mathrm{u} \mathrm{c} \mathrm{h} \mathrm{i}{ }^{27}$ gemessenen Wirkungsquerschnitt für die Sternintensität in Luft ( $h$, in Abb. 6) ausreichen; zum anderen muß die Multiplizität, die die Photonen im Rahmen einer Kaskade in Blei bzw. Eisen gemäß der Kaskadentheorie erfahren, mit der beobachteten Vermehrung der Sternhäufigkeit $\left(h_{2} / h_{1}\right.$ in Abb. 6) im Einklang sein. Der den Photonen auf Grund dieses Ansatzes zukommende Anteil an der Sternintensität ist als Kurve b in den Abb. 2 und 3 eingetragen. Ihre Form entspricht dem mit Zählrohren gemessenen 1. RossiMaximum von Elektron-Photon-Schauern ${ }^{36,37}$.

Die durch Photonen gebildete Sternhäufigkeit betrage also $1 / 10$ der Gesamtzahl $=3,5 \cdot 10^{-6}$ Sterne/ g sec. In Luft reicht das Spektrum der Photonen bis zu $10^{8} \mathrm{eV}$ herab. Photonen geringerer Energie kommen mit vergleichbarer Intensität nicht vor. Von $1-3 \cdot 10^{8} \mathrm{eV}$ ist der Wirkungsquerschnitt für Sternerzeugung nach Tab. 3 von der Energie abhängig. Wir rechnen mit einem mittleren Wirkungsquerschinitt von $Q=5 \cdot 10^{-27} \mathrm{~cm}^{2}$. Um die angegebene Sternhäufigkeit $Z_{\text {phot }}$ zu gewinnen, braucht man einen allseitigen Photonenstrom

$$
I=\frac{Z_{\text {phot }}}{N Q}=7,0 \cdot 10^{-2} \mathrm{~cm}^{-2} \mathrm{sec}^{-1} .
$$

36 P. M. S. Blackett u. B. Rossi, Rev. mod. Physics 11, 277 [1939].

37 J. E. Morgan u. W. M. Nielsen, J. Franklin Inst. 226, 617 [1937]. 
$N$ bedeutet die Zahl der Kerne in $1 \mathrm{~g}$ der photographischen Emulsion $=10^{22} \mathrm{~g}^{-1}$. Das so berechnete $I$ ist das 2,6-fache der allseitigen Elektronenintensität in $3000 \mathrm{~m}$ Höhe (vgl. R o s s i ${ }^{15}$ ). Die empirisch nicht bekannte Zahl der Photonen läßt sich auf Grund der Kaskadentheorie aus der Zahl der Elektronen bestimmen. Das Verhältnis ist abhängig von der Ausbildung der Kaskade, wobei durchweg die Photonenzahl größer ist, und zwar wenigstens um den Faktor 1,5 ${ }^{38}$.

Die beim Übergang von Luft in Blei oder Eisen durch Zählrohre oder - über die Sterne als Indikator - in Photoplatten registrierte Zunahme der Pho-

$$
\uparrow G\left(k_{0}, k_{s}, l\right)
$$

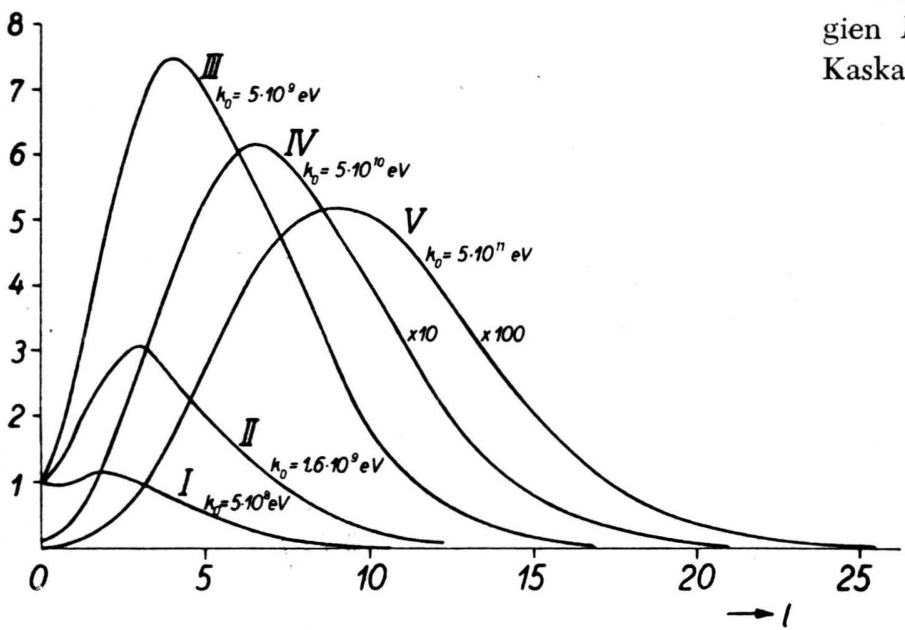

Abb. 8. Berechnete Photonenkaskaden für verschiedene Anfangsenergie $E_{0}$ des Photons.

tonen wird bekanntlich bewirkt durch die Reduktion der unteren Grenzenergie $E_{\mathrm{j}}$, bis zu der sich eine Kaskade ausbildet. Es ist

$$
\begin{aligned}
E_{\mathrm{j}}^{\mathrm{C}}=E_{\mathrm{j}}^{\mathrm{Luft}} & =100 \mathrm{MeV}, \\
E_{\mathrm{j}}^{\mathrm{Fe}} & =26 \mathrm{MeV}, \\
E_{\mathrm{j}}^{\mathrm{Pb}} & =7 \mathrm{MeV} .
\end{aligned}
$$

Dabei ist vorausgesetzt, daß das registrierende Instrument auf Photonenenergien $=E_{\mathrm{j}}$ anspricht. Bei Zählrohren ist das immer der Fall, bei der Photoplatte ist jedoch zur Sternauslösung eine Energie von mindestens $E_{\mathrm{St}}=50 \mathrm{MeV}$ notwendig. Daher wird für die Sternauslösung derjenige Teil der Kaskaden in Blei

38 L. J a n o s s y, H. M e s s e 1, Proc. Roy. Irish Acad., Sect. A 54, 15, 217 [1951].

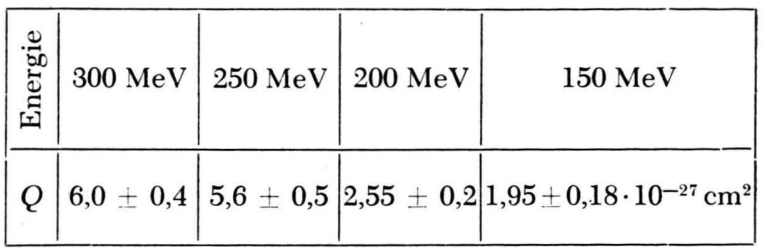

Tab. 3. Wirkungsquerschnitt $Q$ von Photonen für Sternauslösung in der photographischen Emulsion nach $\mathrm{Ki}$ ku ch i 27.

und Eisen abgeschnitten, der unterhalb $E_{\mathrm{St}}$ liegt. Die von der Photoplatte über Photonensterne registrierten Kaskaden in Blei oder Eisen sind somit, wenn man sie über Strahlungslängen aufträgt und von der Winkelstreuung absieht, gleich.

In Abb. 8 ist die Häufigkeit der Photonen mit Energien $E>E_{\mathrm{St}}=5 \cdot 10^{7} \mathrm{eV}$, wie man sie gemäß der Kaskadentheorie bei Auslösung durch ein Photon der

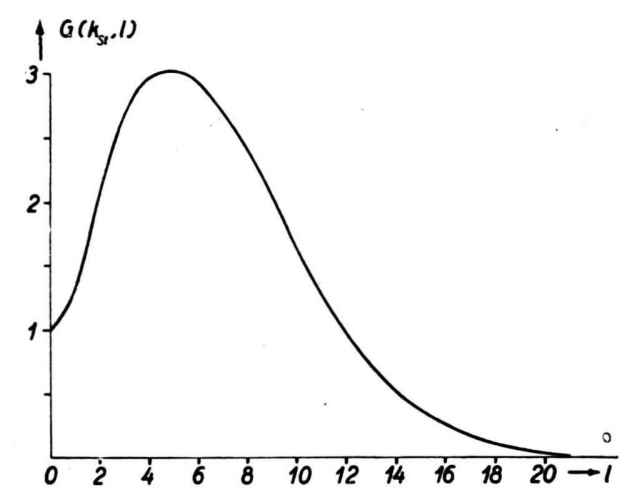

Abb. 9. Berechnete Photonenkaskade für ein Energiespektrum der einfallenden Photonen.

Energie $E_{0}$ erwarten sollte, für verschiedene $E_{0}$ gezeichnet. Der Energieverlust durch Ionisation ist nach dem Verfahren von $\mathrm{Bh}$ a b b a und $\mathrm{Ch}$ a krabarty berücksichtigt (vgl. die Tabellen von J a n o s s y und Messe 1 ${ }^{38}$ ). Die beobachtete Kaskade stellt eine Überlagerung von verschiedenen derartigen Kurven gemäß dem Energiespektrum der auftreffenden Photonen dar. Úber dieses wissen wir heute folgendes: Die Photonen stehen mit der nuclearen Komponente $\left(E_{\mathrm{N}} \geqq 10^{9} \mathrm{eV}\right)$ im Gleichgewicht, da sie fast ausschließlich durch den Zerfall des neutralen $\pi$-Mesons entstehen und ihre Strahlungslänge in allen Materialien kürzer ist als die Absorptionsweglänge der Nucleonen. Das Energiespektrum der Photonen ist daher bestimmt durch das Energiespektrum der sie erzeugenden Nucleonen einerseits und den Erzeugungsmechanismus andererseits. 
In dem uns interessierenden Bereich mit $E>$ $5 \cdot 10^{7} \mathrm{eV}$ gilt ${ }^{39}$ :

Im Gebiet zwischen 200 und $2000 \mathrm{MeV}$ fällt das differentielle Photonenspektrum mit $E^{-(1,6 \pm 0,2)}$ ab. Das ist in großen Höhen für den Bereich von 200 bis $800 \mathrm{MeV}$ von $\mathrm{Carls}$ on und Mitarbeitern ${ }^{12} \mathrm{e}$ gemessen. Da der Exponent in diesem Energiebereich im wesentlichen vom Entstehungsmechanismus abhängt, ist dieses Meßergebnis auch für $3000 \mathrm{~m}$ Höhe verbindlich. Im Bereich extrem hoher Photonenenergien $\left(E>10^{10} \mathrm{eV}\right)$ ist der Exponent des Photonenspektrums gleich dem des Primärspektrums.

Wir setzen daher für das differentielle Photonenspektrum folgendes an:

$$
\begin{array}{ll}
g(E) \sim E^{-1,6} & \text { für } 1 / 2 \cdot 10^{8}<E<10^{10} \mathrm{eV}, \\
g(E) \sim E^{-2} & \text { für } \quad 10^{10}<E<10^{11} \mathrm{eV}, \\
g(E) \sim E^{-2,6} & \text { für } \quad 10^{11}<E .
\end{array}
$$

Entsprechend diesem Spektrum wird die zu erwartende Photonenkaskade in Eisen und Blei für Quanten mit $E>5 \cdot 10^{7} \mathrm{eV}$ berechnet. Das Ergebnis ist in Abb. 9 dargestellt: Die in das Blei oder Eisen eintretende Photonenintensität ist im Maximum verdreifacht.

Diese Aussage hängt vom Energiespektrum der Photonen ab. Für einen Anstieg auf rund das Vierfache, wie es unsere qualitative Analyse ergibt, müßte die Zahl der energieärmeren Photonen $\left(E<5 \cdot 10^{8} \mathrm{eV}\right)$ sehr viel seltener sein, als wir ansetzten. Das widerspricht aber sowohl den Messungen von Carls on ${ }^{12 \mathrm{e}}$ wie den Rechnungen von $\mathrm{O}$ e h m e ${ }^{39}$.

Die Differenz verschwindet, wenn wir die im Blei über neutrale $\pi$-Mesonen erzeugten Photonen mit berücksichtigen. Diese können wir folgendermaßen berechnen. Die Zahl der Nucleonen mit einer Energie zwischen $E$ und $E+\mathrm{d} E$ an der Stelle $x^{\prime}$ sei

$$
h\left(E, x^{\prime}\right) \mathrm{d} E=\gamma H_{0} E^{-\gamma-1} e^{-a x^{\prime}} \mathrm{d} E .
$$

Wir beschränken uns auf relativistische Nucleonen, da nur diese neutrale Mesonen und über deren Zerfall Photonen in nennenswertem Ausmaß erzeugen können. Wir setzen $\gamma=1 ; a^{-1}=310 \mathrm{~g} / \mathrm{cm}^{2}$ Blei. Die Wahrscheinlichkeit für eine Kernwechselwirkung unter Mesonenerzeugung ist: $N_{\mathrm{L}} / A \Sigma_{\mathrm{K}} \mathrm{d} x\left(N_{\mathrm{L}}=\right.$ Loschmidtsche Zahl, A Massenzahl, $\Sigma_{\mathrm{K}}$ Wirkungsquerschnitt je Kern). Die Zahl der entstehenden Mesonen ist nach $\mathrm{H}$ e is e $\mathrm{n}$ b e r ${ }^{40}$ etwa proportional $E^{1 / 2}$; ein

39 R. O e h m e, Z. Physik 129, 573 [1951].

40 W. H e i s e n b e r g, Z. Physik 126, 569 [1949].
Drittel davon ist neutral. Sie erzeugen bei ihrem Zerfall je 2 Photonen. Die Zahl der entstehenden Photonen ist somit gleich der Zahl $n$ der geladenen Mesonen. Wir schreiben

$$
n=0,25\left(\frac{E}{M c^{2}}\right)^{1 / 2} .
$$

Der Zahlenfaktor 0,25 ist an die Messungen von C a m e r in i und Mitarbeiter ${ }^{12 d}$, die eine Erzeugung von $0,6 \pi$-Mesonen je Stern angeben, adjustiert. Die Gesamtzahl der an der Stelle $x^{\prime}$ entstehenden Photonen ist dann

$$
\begin{gathered}
G\left(x^{\prime}\right) \mathrm{d} x^{\prime}=\int_{E=M c^{2}}^{\infty} h\left(E, x^{\prime}\right) \mathrm{d} E \cdot 0,25\left(\frac{E}{M c^{2}}\right)^{1 / 2} \\
\cdot \frac{N L}{A} \Sigma_{K} \mathrm{~d} x^{\prime}=H\left(x^{\prime}\right) \frac{\gamma}{\gamma-1 / 2} \frac{N L}{A} \Sigma_{K} 0,25 \cdot \mathrm{d} x^{\prime} \\
=3,7 \cdot 10^{-3} H\left(x^{\prime}\right) \mathrm{d} x^{\prime},
\end{gathered}
$$

dabei ist

$$
H\left(x^{\prime}\right)=\int_{E=M c^{2}}^{\infty} h\left(E, x^{\prime}\right) \mathrm{d} E .
$$

Die Wahrscheinlichkeit, diese Photonen - gemäß der Kaskadentheorie vermehrt - an der Stelle $x>x^{\prime}$ anzutreffen, läßt sich näherungsweise berechnen, wenn wir mit der Funktion $f(x)$ multiplizieren, die die Kaskadenmultiplikation für das erzeugte Spektrum repräsentiert; die Funktion ist in Abb. 9 dargestellt. Wir haben dann

$$
\begin{aligned}
G(x) & =3,7 \cdot 10^{-3} \int_{x^{\prime}=0}^{x} H\left(x^{\prime}\right) f\left(x-x^{\prime}\right) \mathrm{d} x^{\prime} \\
& \approx 3,7 \cdot 10^{-3} H(x) \int_{x^{\prime}=0}^{x} f\left(x^{\prime}\right) \mathrm{d} x^{\prime} .
\end{aligned}
$$

Die Intensität dieser $\pi^{0}$-Photonen ist ungefähr um einen Faktor 2 unsicher. Der Verlauf von $G(x)$ ist in Abb. $3 \mathrm{a}$ und $4 \mathrm{a}$ für Blei und Eisen dargestellt.

Die beobachteten b-Maxima werden durch Überlagerung der gerechneten Kurven bezüglich der Höhe gut wiedergegeben. In bezug auf die Lage ist die Übereinstimmung etwas weniger gut. Sie ist auch nicht zu erwarten, da die Winkelstreuung der Elektronen in unseren Rechnungen nicht berücksichtigt wurde. Derselbe Effekt tritt übrigens auch bei Schauermessungen mit Zählrohren ${ }^{36,37}$ auf.

Der eingangs gemachte Ansatz, daß 10\% der Sterne in Luft durch Photonen ausgelöst seien, entspricht sowohl dem gemessenen Wirkungsquerschnitt für Sternerzeugung durch Photonen als auch ihrer Vermehrung nach der Kaskadentheorie. Die beobach- 
teten b-Maxima der Sternhäufigkeit in Blei und Eisen stellen Übergangseffekt der Photonenkomponente dar.

Bei der experimentellen Durchführung der Arbeit haben Frl. E. B a r t s c h a t, die Herren G. K u h n, W. B r a u n, E. W e b e r, O. E b e r t wertvolle Hilfe geleistet. Zu besonderem Dank ist der eine von uns (E.Sch.) der Fa.
E. Leitz, Wetzlar, und Herrn Professor B e rg man n für die Überlassung eines zusätzlichen kompletten Ortholux-Mikroskopes mit Spezial-Zubehör, und für die Entwicklung besonderer Kernspur-Objektive verpflichtet. Ferner dankt er der Notgemeins chaft der Deutschen Wissenschaft für eine seit dem Jahre 1938 zur Auswertung von Kernspurplatten überlassene Mikroskop-Ausrüstung.

\title{
Solare Ultrastrahlung und Erdmagnetfeld
}

\author{
Von ARNulf Schlüter \\ Aus dem Max-Planck-Institut für Physik, Göttingen \\ (Z. Naturforschg. 6 a, 613-618 [1951]; eingegangen am 24. August 1951)
}

\section{Herrn Professor E rich Rege ner zum 70. Geburtstag}

\begin{abstract}
Durch numerische Integration von 20 Bahnen geladener Teilchen im Felde eines magnetischer Dipols wurde das Netz der von S törmer berechneten Bahnen, die von der im geomagnetischen Äquator angenommenen Sonne kommen, für den interessierenden Impulsbereich von einigen $10^{9} \mathrm{eV} / c$ soweit verfeinert, daß einige quantitative Schlüsse über die theoretische Verteilung der Ultrastrahlung auf der Erde gezogen werden können. Es ergibt sich in mittleren Breiten ein ausgeprägtes Maximum gegen $9 \mathrm{~h}$ Ortszeit (für positive Teilchen) und ein verbotener Bereich in der Nähe der Pole. Durch Näherungsbetrachtungen, die durch die Ergebnisse der numerischen Rechnung begründet werden, werden die Schlüsse auf noch niedrigere Energien und auf andere Deklination der Sonne ausgedehnt. Ein Teil cler experimentellen Befunde bei den beobachteten solaren Ultrastrahlungsausbrüchen steht in Widerspruch zu den Erwartungen nach der Störmerschen Theorie.
\end{abstract}

$\mathrm{D}$ ie zuerst von Forbush und Ehmert gemessenen und später von zahlreichen anderen Beobachtern bestätigten Anstiege der Intensität der Ultrastrahlung in See- und Berghöhe, die gleichzeitig oder kurz nach chromosphärischen Eruptionen auf der Sonne auftraten, haben sichergestellt, daß die Sonne mindestens zeitweise Ultrastrahlung erzeugt. Hierdurch hat die Frage nach den Bahnen geladener Teilchen, die von der Sonne her auf die Erde treffen, erneut an Interesse gewonnen.

Wir nehmen an, daß das Erdmagnetfeld sich durch ein Dipolfeld vom Momente $M=8,4 \cdot 10^{25} \mathrm{~cm}^{5 / 2} \mathrm{~g}^{1 / 2}$ $\mathrm{sec}^{-1}$ genügend genau annähern läßt. Dann können wir aus der Tatsache, daß bisher noch kein solcher Ultrastrahlungsanstieg am geomagnetischen Äquator beobachtet ist (nämlich in Huancayo, Peru, geomagnetische Breite $\Phi=0,6^{\circ} \mathrm{S}$ ), eine obere Grenze für den interessierenden Impulsbereich der solaren Partikel angeben. Einfach geladene Teilchen ${ }^{1}$ von einem Impuls $P>10,4 \cdot 10^{9} \mathrm{eV} / c \quad(c=$ Lichtgeschwindigkeit $)$ können den Äquator erreichen, und solche mit einem Impuls $P>15,5 \cdot 10^{9} \mathrm{eV} / c$ können dort senkrecht ein-

1 Wir werden im folgenden immer annehmen, daß die Teilchen einfach geladen sind. fallen. Man wird daher annehmen, daß die solaren Ultrastrahlungsteilchen einen Impuls von weniger als $10^{10} \mathrm{eV} / c$ besitzen. Andererseits folgt daraus, daß diese Teilchen oder ihre Folgeprodukte in Seehöhe überhaupt beobachtet werden können, daß sie sicher einen Impuls von einigen $10^{9} \mathrm{eV} / c$ besessen haben.

Nun hat S tö r m e r ${ }^{2}$ bereits im Jahre 193672 Bahnen ${ }^{3}$ von Teilchen berechnet, die im Unendlichen auf dem geomagnetischen Äquator mit verschiedenen Drehimpulsen starten. Da Bahnen verschiedenen Impulses durch eine Transformation des Längenmaßstabes ineinander übergeführt werden können, genügt es, sie für einen bestimmten Impuls zu berechnen. Zweckmäßig ist es dabei, alle Längeneinheiten in der Störmerschen Einheit $\sqrt{e M / c P} \quad(e=$ Elementarladung) zu messen, und statt der Zeit bei der Bahnberechnung die Bogenlänge in diesen Einheiten als Parameter zu benutzen. Für Teilchen großen Impulses $P$ wird die Störmersche Länge klein, der Radius der Erde in Störmerschen Einheiten gemessen $(A)$ also groß. Es gilt die Beziehung

$$
P=A^{2} \cdot 62,1 \cdot 10^{9} \mathrm{eV} / c \text {. }
$$

\footnotetext{
2 C. S t ö r m e r, Astrophysic. Norv. 2, 1 [1936].

3 Wegen der Symmetrie zum geomagnetischen Äquator entspricht dies insgesamt 132 Bahnen.
} 